\title{
Electrochemical Microscopy Based on Spatial Light Modulators: A Projection System to Spatially Address Electrochemical Reactions at Semiconductors
}

\author{
Yan B. Vogel, ${ }^{1}$ Vinicius R. Gonçales, ${ }^{2}$ J. Justin Gooding, ${ }^{2, z}$ and Simone Ciampi ${ }^{1, z}$ \\ ${ }^{1}$ Department of Chemistry, Curtin University, Bentley, Western Australia 6102, Australia \\ ${ }^{2}$ School of Chemistry, Australian Centre for NanoMedicine, ARC Centre of Excellence in Convergent Bio-Nano \\ Science and Technology, The University of New South Wales, Sydney, New South Wales 2052, Australia
}

\begin{abstract}
Here we describe a "light projector" system that can address, i.e. "read and write" electrochemical reactions on a non-structured macroscopic semiconducting electrode with spatial and temporal resolution. In our approach the illumination of an amorphous silicon electrode/electrolyte interface is spatially defined by means of a ferroelectric micromirror system that gives total freedom on both the two-dimensional light profile (illumination shapes) as well as on the transient times of the projected images. The device has no moving parts and allows for spatial and temporal control of the illumination stimulus driving local changes to the rate of an electrochemical reaction. The performance of the system is assessed by generating microscale patterns of $\mathrm{Cu}_{2} \mathrm{O}$ on the electrode ("electrochemical writing") followed by their 2D current mapping ("electrochemical reading") using methanol electro-oxidation and carbon dioxide electro-reduction. The latter illustrate the electrochemical imaging aspects of the device using two technologically relevant examples.

(C) The Author(s) 2017. Published by ECS. This is an open access article distributed under the terms of the Creative Commons Attribution 4.0 License (CC BY, http://creativecommons.org/licenses/by/4.0/), which permits unrestricted reuse of the work in any medium, provided the original work is properly cited. [DOI: 10.1149/2.0111804jes]

(cc) BY
\end{abstract}

Manuscript submitted October 19, 2017; revised manuscript received December 12, 2017. Published December 30, 2017. This paper

is part of the JES Focus Issue on Processes at the Semiconductor-Solution Interface.

The field of spatial localization of electrochemical reactions at interfaces has made tremendous improvements over the last three decades, and recent conceptual and technical advances in the fields of catalysis, energy and sensing have been linked to our ability to quantify adventitious or deliberate two-dimensional inhomogeneity of an electrified interface. ${ }^{1-5}$ Addressing an electrochemical reaction with spatial resolution was originally done by the construction of individually-addressable electrochemical arrays, where each electrode is individually connected to an external power source or measuring instrument. ${ }^{6}$ These arrays use considerable space for the pad connections and the geometry is pre-defined and restricted by the array design, giving limited flexibility to the experimentalist. Moreover, the construction of individually-addressable electrochemical arrays is an expensive and laborious task. One strategy to overcome this problem has been implemented by using a single ultramicroelectrode tip that is mounted on a travelling stage. This is the so-called scanning electrochemical microscopy (SECM), where an ultramicroelectrode is scanned over the sample and without any geometrical restriction. Since the SECM invention, ${ }^{7}$ other scanning electrochemical probe techniques have been developed and used extensively in research, with notable examples being scanning electrochemical cell microscopy, SECCM ${ }^{8}$ and scanning photoelectrochemical microscopy, SPECM. ${ }^{5}$ These scanning techniques are very powerful instruments, both for surface characterization as well as modification. ${ }^{4}$ For instance, electrochemical scanning techniques can now claim spatial resolution down to few nanometers ${ }^{2}$ and imaging speed as high as few seconds per frame. ${ }^{8}$ However, scanning electrochemical techniques present the intrinsic characteristics, and to a degree a limitation, of a scanning probe method. They are "sequential" methods, i.e. only one discrete area can be addressed at a certain time, with the moving tip possibly generating convective forces in the electrolyte. ${ }^{9}$ Furthermore, the investigating ultramicroelectrode "probe" is approaching the electrode from the electrolyte side, hence for instance we can only investigate charge transfer kinetics at an electrified surface indirectly from the electrolyte "point of view". This is because scanning electrochemical techniques have a critical requisite of a one-electrode/one-wire, i.e. it is only possible to turn-on the entire conductive interface and then raster the probe to a specific site.

zE-mail: simone.ciampi@curtin.edu.au; justin.gooding@unsw.edu.au
One could envision a simplification of these rather sophisticated electrochemical imaging techniques by shifting the tenet of oneelectrode/one-wire toward one-wire/many-electrodes, doing so by turning an insulator into a transient conductor only at one specific site that is defined by the experimentalist. Some of us ${ }^{10-12}$ and others ${ }^{13-17}$ have recently started making use of semiconductor electrodes to drive redox processes in a spatial-selective fashion. In this context, Choudhury et al. recently reported a strategy to spatially confine an electrochemical reaction using the intrinsic semiconducting property of silicon to generate and separate charge carriers when illuminated. ${ }^{10}$ These charge carriers oxidize or reduce nearby species present in the electrolyte or tethered to the surface. Directing the flux of carriers on the surface is a matter of controlling the illuminated areas, as schematized in Figure 1a. As a proof-of-principle experiment, Choudhury et al. used a light pointer mounted on a travelling $x-y$ stage to guide the illumination in 2D. However, to fully exploit the potential of this "light-addressable" electrochemistry concept it is necessary to develop a method that allows to control the time and shape of the illumination with greater freedom, therefore using an illumination source akin of a microscale projector.

Here we describe an optical/electrochemical instrument (see Figure 1d for a picture of the set-up) to perform localized electrochemical reactions with a resolution of few micrometers $(4.6 \mu \mathrm{m})$ at a non-structured photoelectrode by this "light addressable" electrochemistry principle. The experimental set-up is composed of a ferroelectric micromirror that allows temporal and spatial control of a light pattern illuminating discrete regions of a semiconducting electrode, a single-channel potentiostat and a conventional single-compartment electrochemical cell (Figures 1 and 2). By generating illumination patterns on an amorphous silicon photoelectrode surface, carriers are excited locally on the illuminated areas (Figures 1a, 1b) allowing to confine electrochemical reactions in space. As a proof-of-principle, patterns of cuprous oxide $\left(\mathrm{Cu}_{2} \mathrm{O}\right.$ hereafter) nanoparticles are formed on the substrate (Figure 1c and Figure 4) by using these carriers to reduce copper (II) ions present in the electrolyte solution in contact with the solid surface. This device is also used for the two-dimensional characterization of charge-transfer reactions on photoelectrodes. To this end the microfabricated substrates with patterns of $\mathrm{Cu}_{2} \mathrm{O}$ nanoparticles were assessed, i.e. "mapped", for the electro-oxidation of methanol and electro-reduction of $\mathrm{CO}_{2}$. 
a)

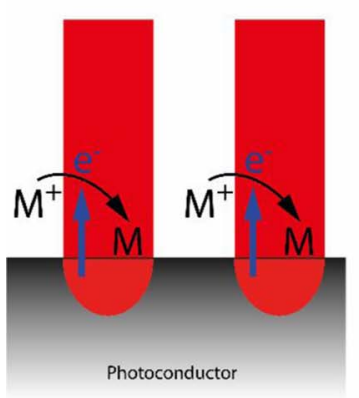

b)

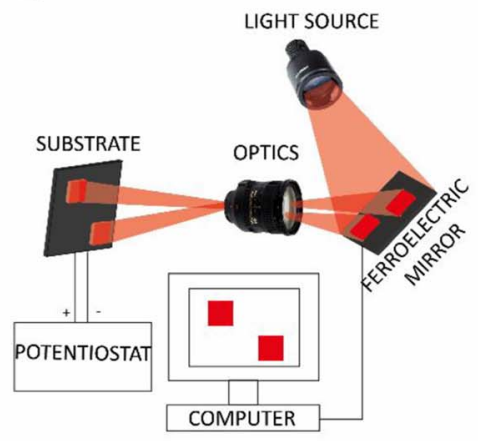

c)

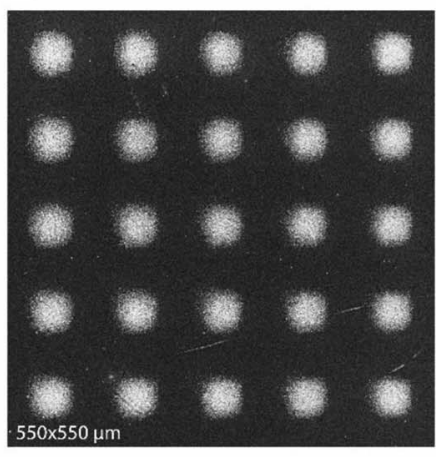

d)

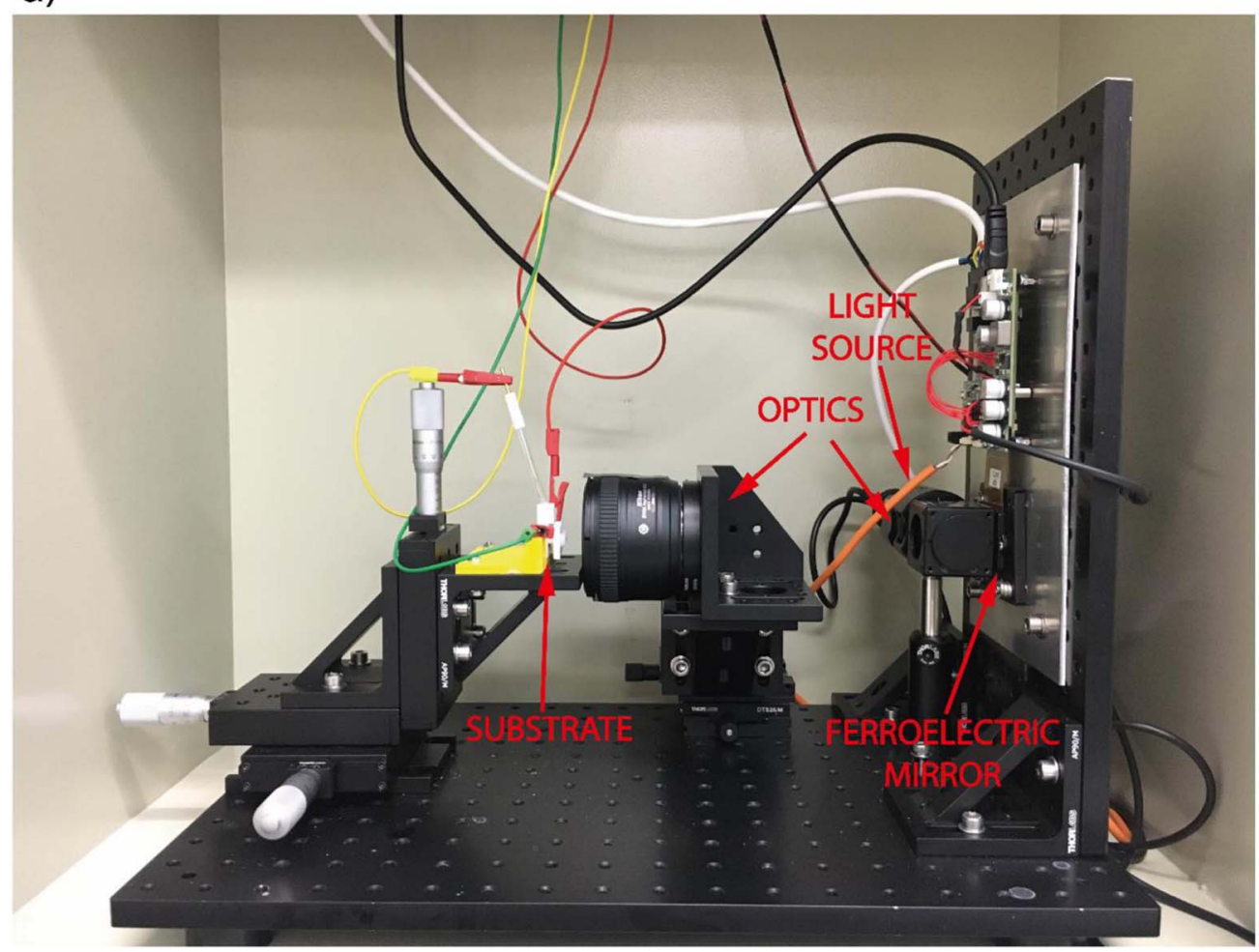

Figure 1. Working principle of the device. (a) Illumination of a photoelectrode generates charge carriers that are used to drive electrochemical reactions, in this case the discharge of metal ions with formation of metallic patterns only on illuminated discrete areas of the electrode surface. (b) Diagram of the set-up. This device allows to control illumination on the photoelectrode hence confining the electrochemical reaction in space and time. This concept can be used in semiconductors or photoconductors, for example, to deposit particle micropatterns by simply projecting on the substrate a specific user-defined illumination pattern. (c) SEM image of a $\mathrm{Cu}_{2} \mathrm{O}$ micropattern deposited using this concept by discharging cupric ions from the electrolyte solution. An array of bright squares $46 \mu \mathrm{m}$ in width and separated by gaps of $92 \mu \mathrm{m}$ was projected on the surface (d) Picture of the actual set-up here described. The electrodes are connected to a general potentiostat which has been omitted for clarity (see Figure S2 in the supplementary material for a picture including the potentiostat).

\section{Experimental}

Overall instrumental design.-The instrument consists of four main parts schematically depicted in Figure 1b: 1) a "microdisplay" that allows spatial and temporal control of the light, which is composed of a light source, a ferroelectric mirror and optics, 2) a singlecompartment, three-electrode cell where the photoelectrode is placed and connected to 3) a single-channel potentiostat, and 4) a computer to control both the microdisplay and the potentiostat. Figure 1d shows a picture of the whole device. Supporting Table S1 is a detailed description of all the components.

Microdisplay.-Light from an LED is projected onto a photoconductive amorphous silicon working electrode after being reflected by a ferroelectric micromirror device that acts as spatial light modulator.
A scheme with the main parts that compose the overall microprojector is shown in Figure 2. The principle of operation is as follows: the light $(\lambda=625 \mathrm{~nm})$ from a LED (1) is collimated by an aspheric lens (2) and then polarized (3). The polarized light beam is then collected by a polarizer beam splitter (4), which allows one polarization to pass undisturbed through it, hence removed from the system, while the other polarization is reflected toward the ferroelectric liquid crystal on silicon (FLCoS (5) controlled via two separate circuit boards (7), model QXGA-3DM from Forth Dimension Displays Ltd. The FLCoS micromirror device is made up from over 3 million $(2048 \times 1536)$ discrete and adjacent $(0.24 \mu \mathrm{m}$ of interspacing) FLCoS elements, each being a square of $7.96 \mu \mathrm{m}$ in width. Each one of these FLCoS elements can be individually controlled to modulate the overall light polarization of the beam through the physical principle of birefringence according to electrically-induced changes of the liquid crystal 
a)
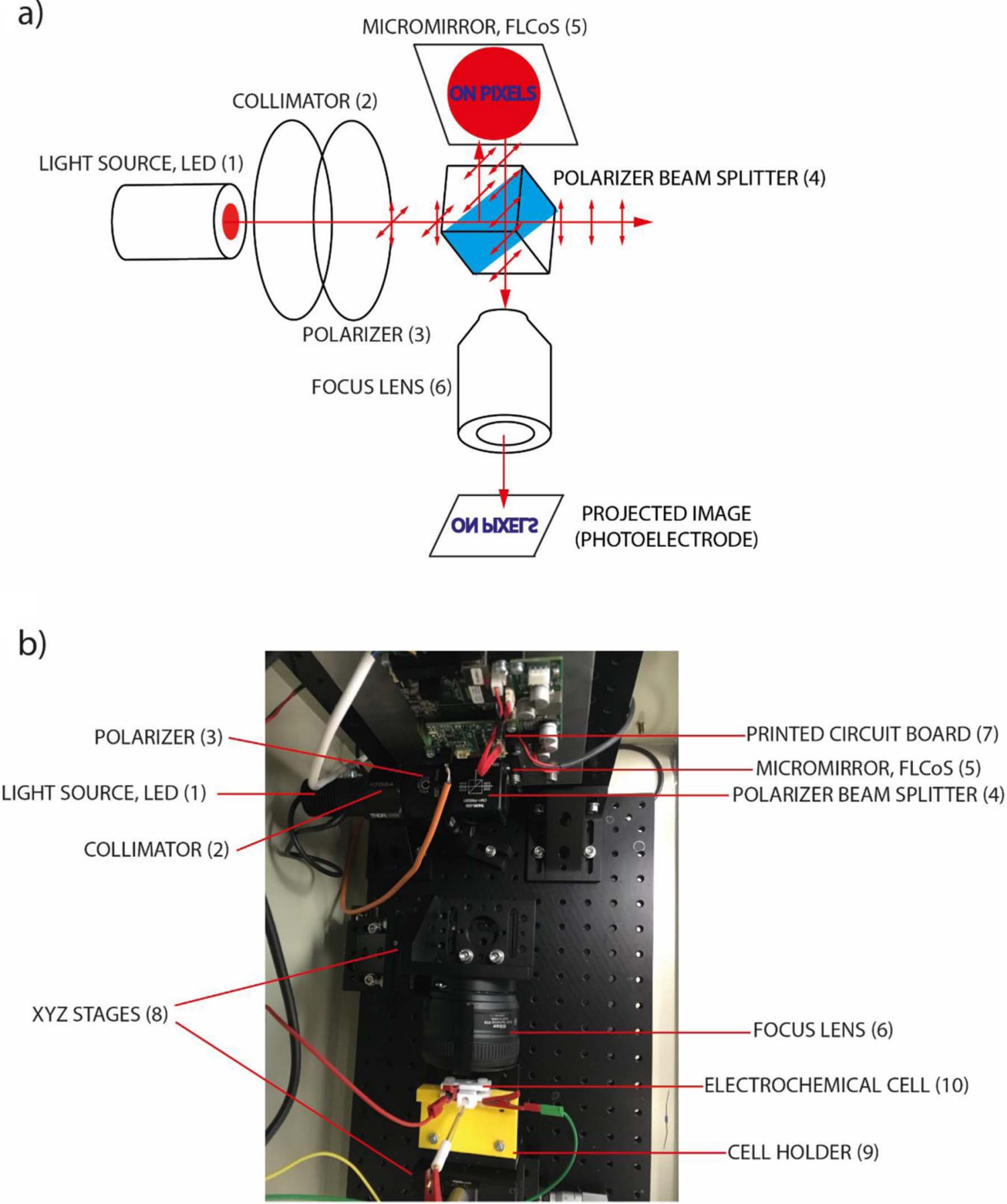

Figure 2. Main components of the microdisplay set-up. (a) Scheme of the main parts comprising the microdisplay. The light is represented as a solid red line with an arrow indicating the direction of travel and perpendicular arrows indicating its polarization. (b) Top-view picture of the microdisplay indicating its main components. Parts are numbered according to Table S1 in the supplementary material.

orientation. This gives to the light reflected by each FLCoS element only two statuses, which can be thought as ON/OFF. For the ON status the polarization is changed by $90^{\circ}$ allowing the beam to go back toward the polarizer beam splitter and to cross it undisturbed. On the other hand, the OFF status of an FLCoS element does not change the polarization and the light is reflected by the polarizer beam splitter and removed from the system. All the ON statuses after crossing the polarizer beam splitter are collected by the focus lens (6) and finally projected onto the substrate situated in a three electrodes electrochemical cell ((9) and (10) in Figure 2 and Figure 3). The focus lens (6) and the cell holder (9) sit on $x-y-z$ traveling stages (8) to allow focusing and/or magnification/reduction of the projected image. To prevent damages to the liquid crystals, the net bias across it must equal zero over time, that is to say each ferroelectric liquid crystal micromirror must be switched ON and OFF intermittently. Because of this, the projected light is intermittent in nature with a frequency that can be modulated in a range between ca. $500 \mathrm{~Hz}$ to ca. $10 \mathrm{kHz}$. This requirement needs an accurate synchronization between the LED light source and the FLCoS micromirror device which is achieved by means of two separate boards (7); one board for the LED driving and the other is 
a)

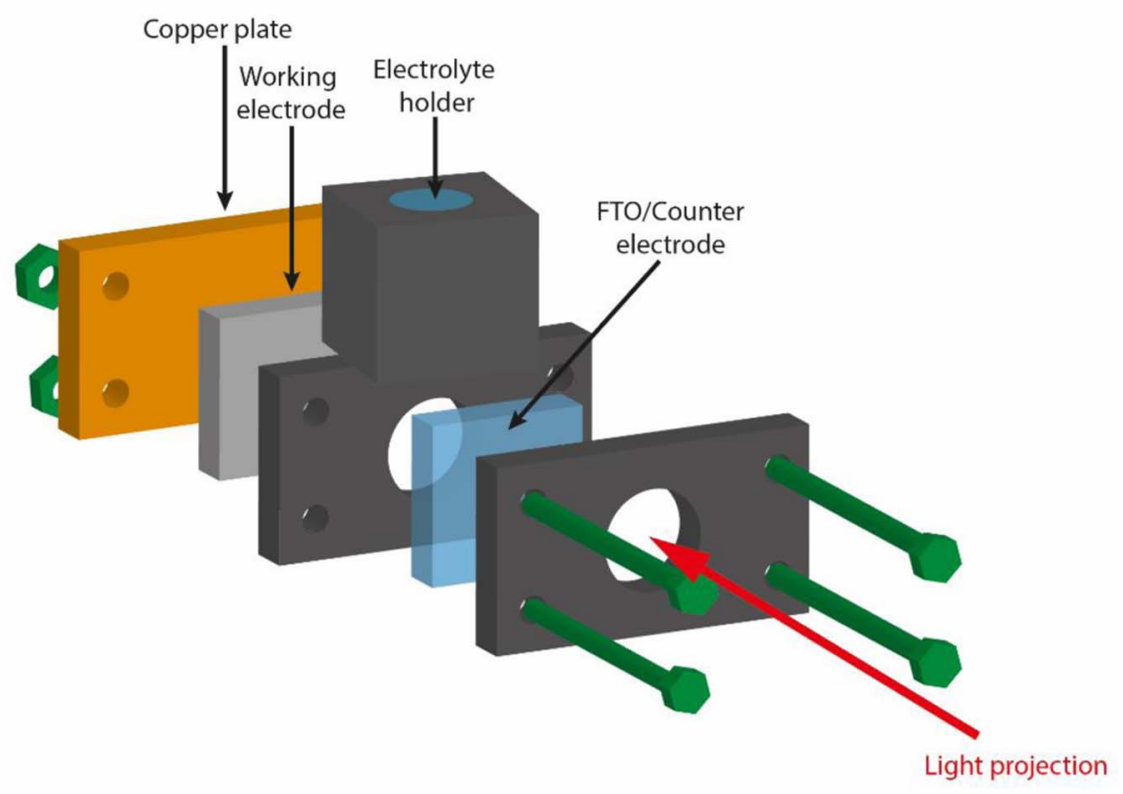

b)

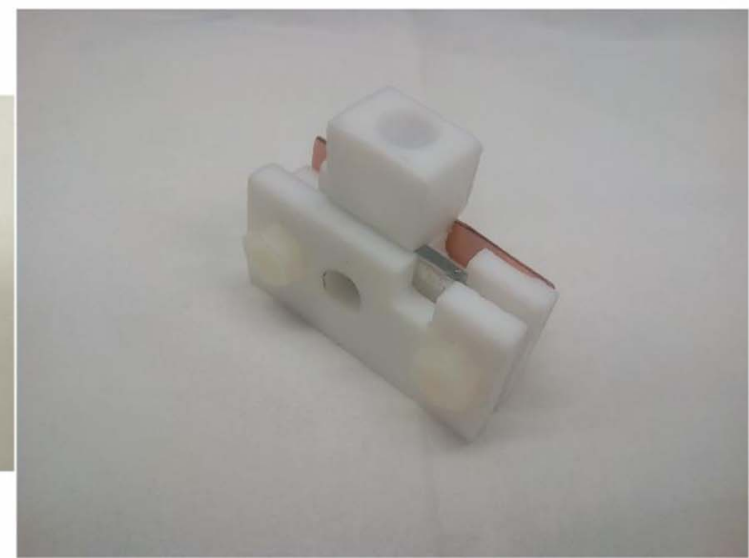

Figure 3. a) Scheme and b) picture of the electrochemical cell. The electrochemical cell was CNC-machined from a PTFE block. Two "o-rings" (not shown in the scheme) are placed between the FTO and electrolyte holder and between the working electrode and electrolyte holder to prevent leaking of the electrolyte.

the microdisplay interface for timing, ordering and synchronization of the images uploaded on its memory.

Electrochemical cell and amorphous silicon substrate preparation and derivatization.-Figure 3 a shows a blown-up scheme of the electrochemical compartment. The electrochemical cell was machined with a 5-axis CNC from a polytetrafluoroethylene (PTFE) block. The geometry of the electrochemical cell was studied to allow the spatiallymodulated light beam coming from FLCoS to be illuminated on the working electrode surface with minor scattering from a liquid/air interface. The light is therefore projected on the working amorphous silicon electrode from the electrolyte side after passing through a conductive layer of FTO deposited on a glass slide. The latter serves also as the counter electrode. The light path through the electrolyte was minimized to a length of ca. $2.5 \mathrm{~mm}$ to avoid as much as possible light intensity absorbance from the electrolyte. $\mathrm{An} \mathrm{Ag}|\mathrm{AgCl}| \mathrm{KCl}$ (sat.) reference electrode is placed in a small reservoir/port sitting just on the top of the working and counter electrodes. This port is also used to inject electrolyte in the cell and remove air bubbles. The electrochemical cell is then fitted on a holder (9) which is mounted tightly on a travelling stage ((8), see Figures 1d and 2) to keep it at an accurate position and/or allowing for precise changes to its position.

As explained in the introduction section, spatially and temporally addressing electrochemical reactions requires a light stimulus as well as a photoconductive substrate, i.e. a substrate that allows electron transfer only when illuminated. For that we used a photoconductive thin layer $(1 \mu \mathrm{m})$ of intrinsic amorphous silicon (a-Si hereafter) deposited on a highly-conductive substrate (a $\mathrm{Si}(100)$ wafer with a resistivity of $0.001-0.004 \Omega \mathrm{cm}$ ). The a-Si layer faces the electrolyte, from where it is illuminated, and the $\operatorname{Si}(100)$ is back-contacted against a copper plate after applying gallium-indium eutectic to ensure an ohmic contact before connecting to the potentiostat circuit. The a$\mathrm{Si}$ thin layer acts as a dark insulator and a photoconductor; only at the illuminated area the conductivity increases to address localized electrochemical reactions.

Amorphous Silicon (a-Si) preparation method.-P-type $\mathrm{Si}(100)$ wafers with a resistivity of $0.001-0.004 \Omega \mathrm{cm}$ (boron-doped) were rinsed with DCM (Ajax Finechem), dried under a stream of argon, and immersed in piranha solution $(3: 1(\mathrm{v} / \mathrm{v})$ mixture of concentrated sulfuric acid $\left(\mathrm{H}_{2} \mathrm{SO}_{4}\right.$, Baker) and $30 \%$ hydrogen peroxide) for $10 \mathrm{~min}$. Samples were then rinsed with Milli-Q water for $10 \mathrm{~min}$ before being transferred to hydrofluoric acid (HF, EMSURE grade, Merck) aqueous solution $\left(1: 10 \mathrm{HF} /\right.$ water $\left.\mathrm{T}=30^{\circ} \mathrm{C}\right)$ for $60 \mathrm{~s}$. Finally, the $\mathrm{Si}-\mathrm{H}$ wafers were rinsed with Milli-Q water for $1 \mathrm{~min}$. Undoped amorphous silicon was then deposited using an Oxford Instruments Plasmalab 100 plasma-enhanced chemical vapor deposition system. $\mathrm{SiH}_{4}$ (Coregas, 99.999\%) was used as the precursor gas and $\operatorname{Ar}$ (Coregas, 99.99999\%) 
a)

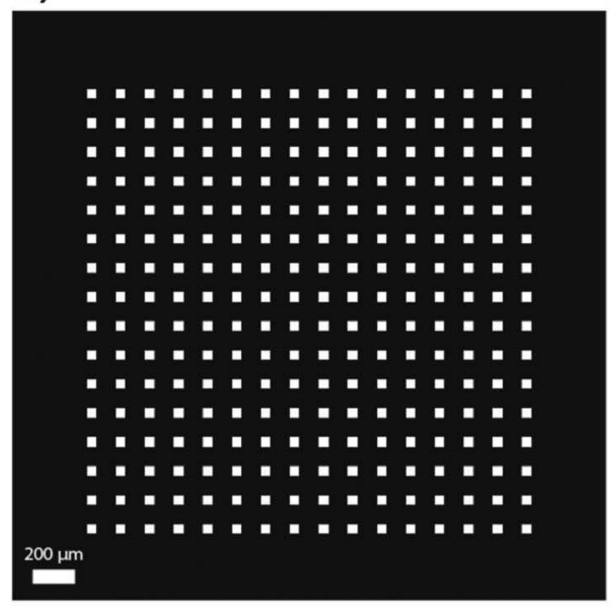

c)

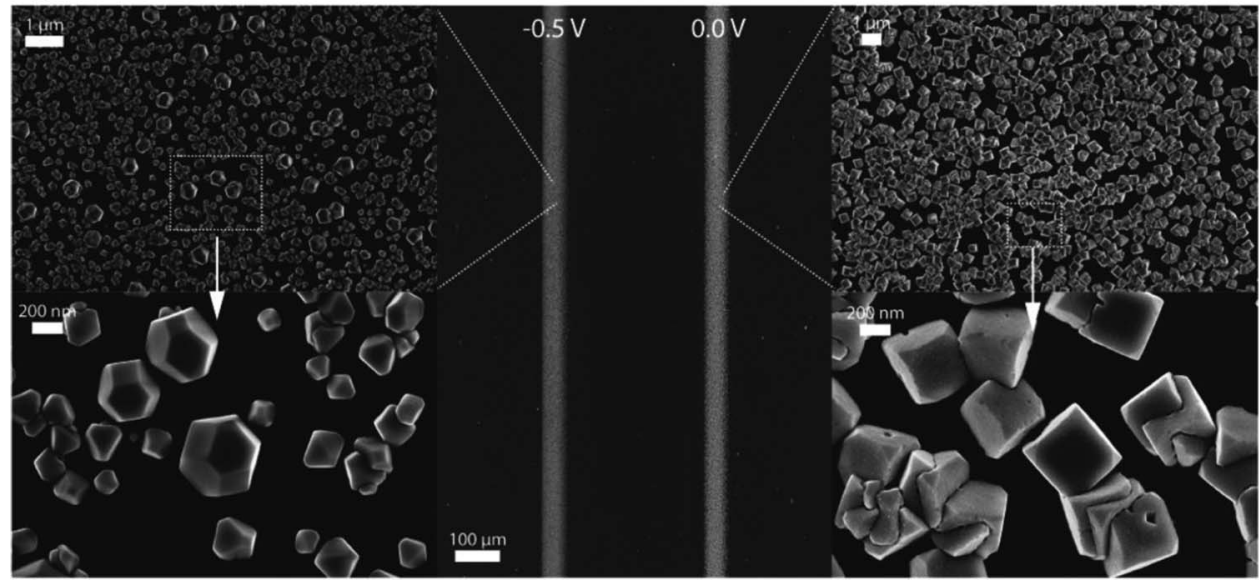

b)

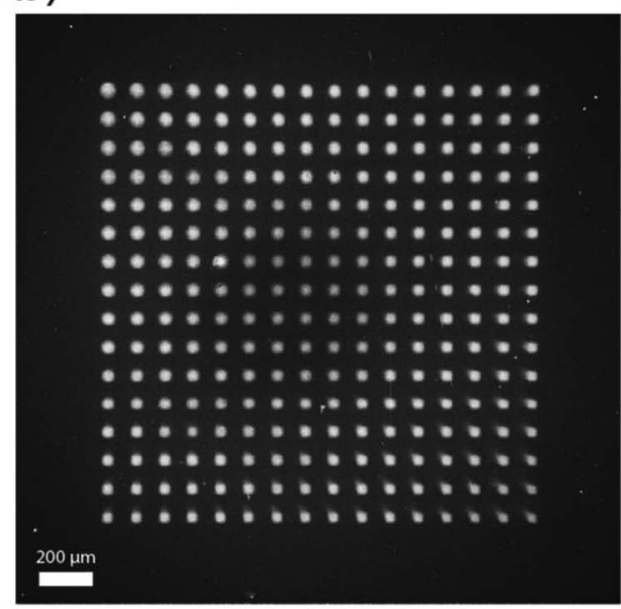


uploaded to the memory on the printed circuit board of the micromirror device (7) using the commercial software MetroCon V3.2 (Forth Dimension Displays) and programmed to be linked to a specific light modulation frequency, time and sequence. The number of images to be uploaded is intrinsically limited by the internal memory, 4 Gbit corresponding with 1024 1-bit images at the given resolution. In this way a movie, i.e. a sequence of images linked to the time each one is projected, is generated. The size of each pixel of the projected image at the magnification here used is $4.6 \mu \mathrm{m}$, which was measured with a travelling microscope by projecting a square of a known number of pixels and then then dividing the length measurement of the square by the number of pixels (known from the uploaded image), hence giving the size of a single projected pixel. Note that a square of $4.6 \mu \mathrm{m}$ is therefore in the current set-up the minimum area the electrochemical reaction can be resolved.

\section{Results and Discussion}

Experimental verification of the device.-In order to experimentally verify the performance of the instrument described above with regards to spatially (2D) resolving electrochemical reactions we selected particle deposition as a proof-of-concept example. The electrochemical reduction of copper (II) ions was used to deposit $\mathrm{Cu}_{2} \mathrm{O}$ particles arranged in microscale patterns on the a-Si working electrode surface. This reaction, according to published work, ${ }^{21}$ takes place via a two-step mechanism with first the reduction of the cupric to cuprous ions, followed by precipitation of $\mathrm{Cu}_{2} \mathrm{O}$ through the following $2 \mathrm{Cu}^{+}+\mathrm{H}_{2} \mathrm{O} \rightarrow \mathrm{Cu}_{2} \mathrm{O}+2 \mathrm{H}^{+}$. X-ray photoelectron spectroscopy experiments shown in Figure $\mathrm{S} 2$ of the supplementary material confirm that the particle composition is $\mathrm{Cu}_{2} \mathrm{O}$. The spatial-temporal control on the light achieved by the set-up described here permits to confine electrochemical reactions to discrete regions of the photoelectrode. By generating light patterns on the electrode surface, such as the one shown in Figure 4a, the electrochemical reduction of copper ions will take place only where the light impinges the surface and the deposition can therefore be spatially addressed. This generates $\mathrm{Cu}_{2} \mathrm{O}$ patterns that accurately follow the projected image. Figure $4 \mathrm{~b}$ shows a deposited pattern of $\mathrm{Cu}_{2} \mathrm{O}$ made by projecting the image of Figure 4a. The electrochemical resolution is somewhat restricted by the dimensions of the projected light when a single element of the FLCoS is turned "ON", which in our system is a $4.6 \mu \mathrm{m}$. When compared with scanning electrochemical techniques that nowadays have resolution of few nanometers ${ }^{2}$ this resolution seems to be quite low; however, there is the theoretical possibility of improving resolution $R$ toward the diffraction limit, which is given by $R=\frac{\lambda}{N A}$, where $\lambda$ is the light wavelength and $N A$ the numerical aperture. This makes it in principle possible to eventually reach a sub-micrometer resolution. As the active element is an array containing more than 3 million of dynamic mirrors, this micropatterning method does not need photomasks and the deposited pattern is built within few seconds, in contrast to sequential deposition methods such as the SECM that need to "scan" to build the pattern or photolithography which relies in the use of photomasks. However sequential deposition, i.e. depositing one specific area after another, is also possible. This is important as depositing patterns sequentially makes possible to control the deposition conditions, e.g. controlling particle morphology, size or density over the substrate by varying the deposition conditions from one region to another. For example, we found that changes in particle "cubicity" are driven by the applied bias; the particle shape changes progressively from cubes to octahedrons as the potential is made more cathodic. ${ }^{a}$ Hence, the

a Traditional views have held that efforts to control the shape of nanocrystals hinge around chemical means. It is known that changes to the concertation of the additive impact the relative growth rate of the different crystallographic faces, hence this impurity/additive can guide the final crystal shape. From SDS, to citrate to halide anions, additives most often carry a net electrical charge. Unpublished results from the authors show that it possible to use near-surface electric fields to modulate the anisotropic interactions between additives and surfaces, hence predictably linking near-surface electric fields to the shape of nanocrystals.

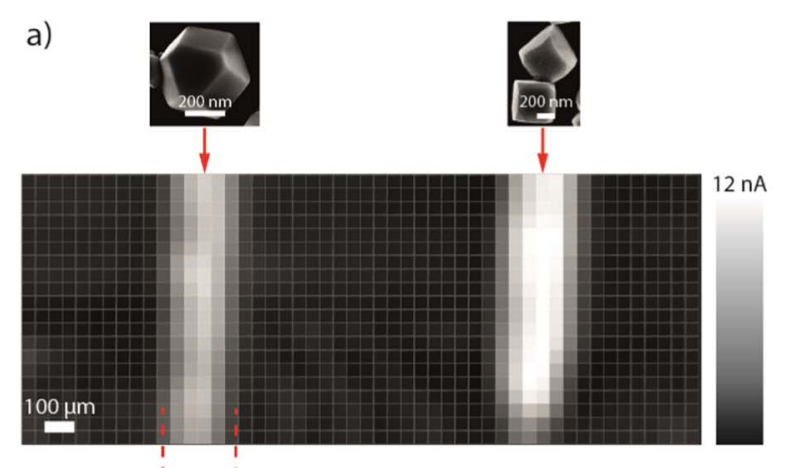

b)

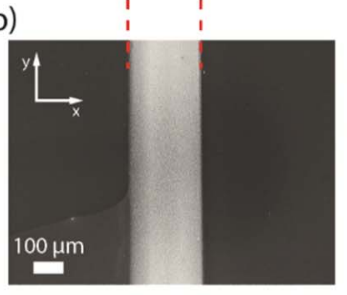

C)

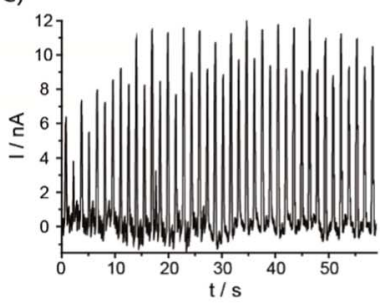

d)

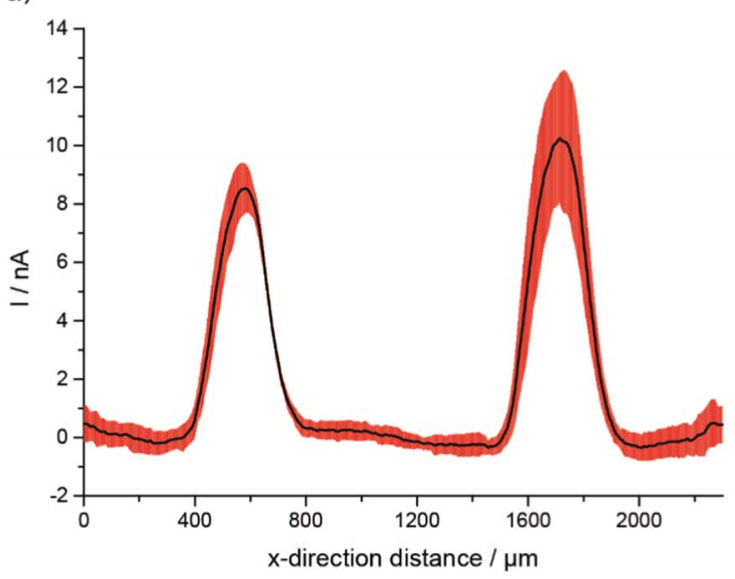

Figure 5. Electrochemical imaging for methanol oxidation. (a) Electrochemical image taken with the device here described for the electrooxidation of methanol at $+0.5 \mathrm{~V}$ (vs $\mathrm{Ag}|\mathrm{AgCl}| \mathrm{KCl}$ (sat.)) by sequentially illuminating square sections of $46 \mu \mathrm{m}$ one next to the other at $17 \mathrm{~Hz}$. Electrolyte is 0.1 $\mathrm{M} \mathrm{NaOH}$ and $0.25 \mathrm{M}$ methanol. Each pixel on the image is $46 \mu \mathrm{m}$ in width. (b) SEM image of the $\mathrm{Cu}_{2} \mathrm{O}$ "line" showing a match in the width with the electrochemical image. Images in panels (a) and (b) are shown at the same scale. (c) Current-time data of the electrochemical image shown in panel (a) before being converted to the bitmap image file. The time $0 \mathrm{~s}$ corresponds with the bottom-left pixel of the electrochemical image in panel (a), and the time $59 \mathrm{~s}$ with the top-right pixel. The current intensity data was averaged every 59 $\mathrm{ms}$ (59 points at $1 \mathrm{~ms}$ sampling interval), corresponding with a single pixel in the electrochemical image. The series of "spikes" in the current transient data correspond to the time when the projected light passes over the $\mathrm{Cu}_{2} \mathrm{O}$ "line", which gives a higher current intensity signal compared to the bare photoconductor. The alternate high and low intensity current "spikes" relates to different activities of cubic vs. octahedral particles. (d) Averaged electrochemical 1D current-distance traces sampled by rastering the light stimulus perpendicular to the lines of $\mathrm{Cu}_{2} \mathrm{O}$ particles ( $x$-direction distance) at different y-distance offsets. The red contour represents the standard deviation.

possibility to grow particles in different areas while changing the deposition conditions makes this technique of interest in research aimed at a systematic screening of factors such as deposition potential on the evolution of crystals' shape. For example, in Figure 4c firstly the left "line" was deposited by projecting a rectangular image, i.e. the "line", while applying a potential of $-0.5 \mathrm{~V}$ vs $\mathrm{Ag}|\mathrm{AgCl}| \mathrm{KCl}$ (sat.) to the entire substrate and stopping this when a charge of $500 \mu \mathrm{C}$ had passed. After this, the right "line" region was illuminated while applying a 
a)

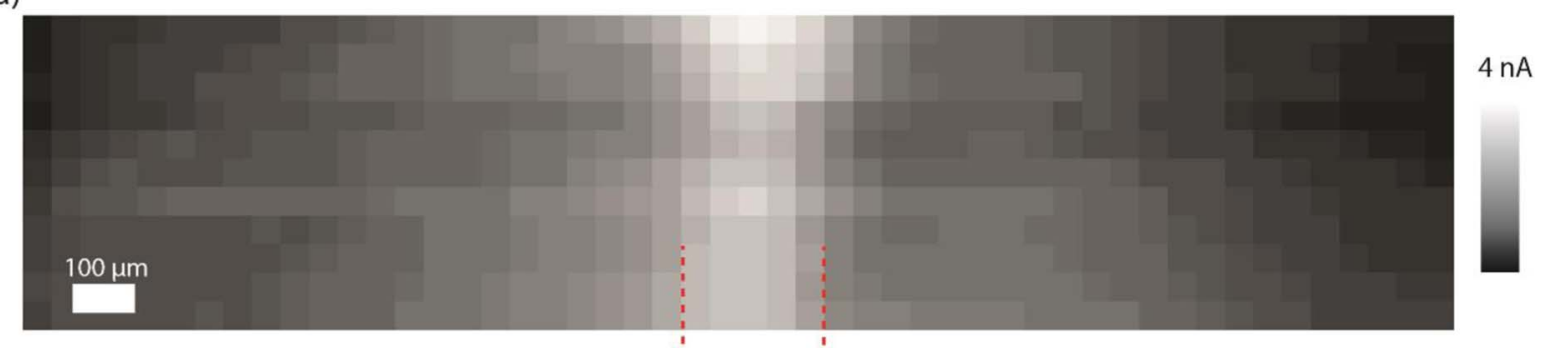

b)

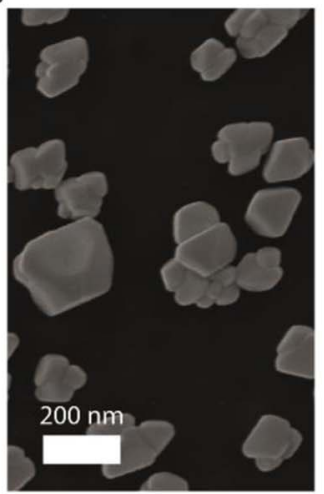

c)

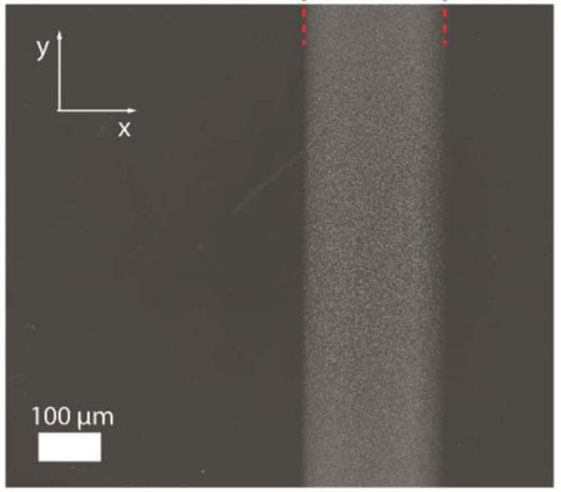

d)

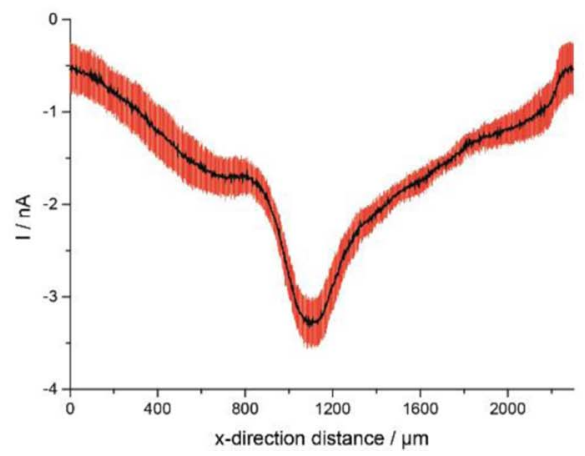

Figure 6. Electrochemical imaging of $\mathrm{CO}_{2}$ reduction. (a) Electrochemical image for the electro-reduction of $\mathrm{CO}_{2}$ by a pattern of $\mathrm{Cu}_{2} \mathrm{O}$ particles taken with the micro projector device here described. The silicon electrode is biased at $-0.5 \mathrm{~V}$ (vs $\mathrm{Ag}|\mathrm{AgCl}| \mathrm{KCl}$ (sat.)) and it is illuminated by projecting sequential images of squares $46 \mu \mathrm{m}$ in side, one next to the other, at a frequency of $17 \mathrm{~Hz}$. Electrolyte is $0.1 \mathrm{M} \mathrm{K}_{2} \mathrm{SO}_{4}$ saturated in $\mathrm{CO}_{2}$. Each pixel on the image is $46 \mu \mathrm{m}$ wide. (b) Magnified SEM of the $\mathrm{Cu}_{2} \mathrm{O}$ particles. (c) SEM image of the $\mathrm{Cu}_{2} \mathrm{O}$ "line", showing a match between the known width (SEM data) and the electrochemical read-out image. (d) One-dimensional current-distance (x-direction) averaged y-direction data (black solid line) and standard deviation (red contour) of the data used to build the electrochemical image shown in panel (a). Images in panels (a) and (c) are shown at the same scale.

potential of $0.0 \mathrm{~V}$ and stopping after reaching the same charge. The only difference on the deposition conditions of the two "lines" is the deposition potential: at more cathodic potentials the electro-deposited particles grow as cuboctahedrons while at less cathodic potentials the particles" grow as cubes. This morphology control is interesting as the catalytic and electronic properties of nanoparticles is in general, and for $\mathrm{Cu}_{2} \mathrm{O}$ in particular, linked to shape. ${ }^{22,23}$

$\mathrm{Cu}_{2} \mathrm{O}$ is known to be a good catalyst for a variety of reactions such as for example methanol oxidation and carbon dioxide reduction. ${ }^{24}$ This aspect is used here to illustrate the "electrochemical reading" potential of the device, that is to say, using a structured and rastering light beam to localize electrical conductivity with microscale resolution to interrogate the electrochemical activity of the surface. Figure 5 a shows an electrochemical image for the methanol oxidation taken on a photoelectrode modified with $\mathrm{Cu}_{2} \mathrm{O}$ particles patterned by the method described above: two "lines" were deposited at two different potentials to obtain cuboctahedral particles (left line) and cubic particles (right line). To construct the electrochemical image from Figure $5 \mathrm{a}$ individual light squares of $46 \mu \mathrm{m}$ in width (equivalent to 10 pixels of the uploaded image) were projected for $59 \mathrm{~ms}$ in rapid sequence, one immediately after the other (corresponding to a frequency of 17 $\mathrm{Hz}$ ). A constant potential of $+0.5 \mathrm{~V}$ vs $\mathrm{Ag}|\mathrm{AgCl}| \mathrm{KCl}$ (sat.) is applied during the whole sequence which takes a total time of $59 \mathrm{~s}$ to record (Figure 5a). The electrochemical image in Figure 5a was generated using the current-time data shown in Figure $5 \mathrm{c}$; the time data was transformed into $(x-y)$ coordinates by simply knowing the amount of time and area each square was projected and associated with the electrochemical current at that specific time-area. Each of the projected squares therefore represent a "pixel" in the electrochemical image. We note that each of the pixels shown in the electrochemical image is formed from a total of 59 averaged data points, as the sample interval used was of $1 \mathrm{~ms}$ and each square was projected for $59 \mathrm{~ms}$. The image resolution is only restricted by the micromirror memory which can store up to 1024 images, corresponding for example to an electrochemical image of $32 \times 32$ pixels. The scan frequency is in principle limited only by the charging time of the double layer.

Remarkably, the electrochemical image that generates from the sample is in very a good agreement with the known physical width of the "lines" as determined by SEM (Figure 5b). Even more interesting, the pattern made up of cubic $\mathrm{Cu}_{2} \mathrm{O}$ particles shows the highest current intensity toward methanol oxidation, a fact that is apparent to the eye of the observer when looking at the electrochemical image in Figure 5a. Figure 5d shows $2 \mathrm{D}$ x-direction current profiles that are generated by "rastering" at different y-direction offsets the light stimuli first across the "line" formed of cuboctahedral particles and then across "line" formed of cubic particles (left to right in Figures 4 and 5) and it reinforces that a higher current intensity is obtained for the cubic-shaped particles. Capacitance measurements (see Figure S3 in the supporting material) suggest this difference in current magnitude is a result of a face-dependent effect rather than due to a difference in the active surface area. This is probably related with the finding of previous reports that assigned a higher catalytic activity to the (100) face of cubic $\mathrm{Cu}_{2} \mathrm{O}$ particles. ${ }^{24,25}$ We remark that having the two patterns under scrutiny (i.e. cubes vs. polyhedron) deposited over the same electrode, exposed to exactly the same electrolyte, $\mathrm{pH}$, same illumination intensity and same cell geometry, removes a great deal of experimental ambiguity when attempting a comparison between catalytic properties of the differently shaped particle deposits. This aspect highlights one of the key advantages of electrochemical microscopy principle delivered by our "micro-projector" design.

Finally, although the data above demonstrate the electrochemical reading capabilities of this device for electro-oxidation reactions we show that electro-reductions are also possible. Figure 6a shows an electrochemical image for the reduction of $\mathrm{CO}_{2}$ on a photoelectrode modified with $\mathrm{Cu}_{2} \mathrm{O}$ particles deposited locally as shown in the SEM image of Figure 6b. As for the data in Figure 5, the dimensions of 
the electrochemical read-out are in good agreement with the known width of the $\mathrm{Cu}_{2} \mathrm{O}$ pattern (Figures $6 \mathrm{c}$ and $6 \mathrm{~d}$ ).

\section{Conclusions}

Here we have described the construction of a device that performs spatially-resolved electrochemical reactions by projecting userdefined light patterns on photoelectrodes. The light generates charge carriers on the photoelectrode allowing the reaction to happen only with an appreciable rate in correspondence of the illuminated area. This device has no moving parts, uses a general one-channel potentiostat and an unstructured surface, giving total freedom on the space and time of the illumination and therefore also on the space and time of theelectrochemical reaction. We have experimentally tested this device for micropatterning of particle deposits on a photoconductor amorphous silicon surface. Microscale patterns can be built very rapidly, without the need of photomasks, by projecting an arbitrary light pattern by means of a dynamic array of FLCoS micromirrors. In particular, we showed this micropatterning capability by electrodepositing patterns of $\mathrm{Cu}_{2} \mathrm{O}$ particles. We also showed that by changing the electrodeposition potential the particle's degree of "cubicity" can be controlled over space. The ability to use light to localize electrochemical reactions with micron-scale resolution can in turn be used to interrogate the electrochemical activity of the surface. We have shown this by spatially resolving the surface electroactivity of $\mathrm{Cu}_{2} \mathrm{O}$ particles toward methanol oxidation and $\mathrm{CO}_{2}$ reduction on an amorphous silicon semiconducting electrode. As the device does not present moving parts this "reading" is done rapidly, where the only practical restriction in terms of imaging speed comes from the charging of the double layer. Patterns of different materials such metals, metal oxides, polymers or molecules could be possibly made on many types of semiconducting or photoconducting surfaces; the device can operate with radiation in the 1 to $3 \mathrm{eV}$ range and accommodate up to 3 wavelengths sources to be used simultaneously. It could be used to test the performance of new electrocatalytic materials for energy conversion (e.g. fuel and solar cells and batteries) or in biological applications (e.g. evaluate the enzymatic activity and detect metabolites of living cells) in an easy and rapid manner. It is important to point out that this device can be used for both surface micropatterning and electrochemical imaging; both in electrochemical reductions (e.g. metal reduction for deposition, reductive electrocatalysis) as well as for oxidative processes (e.g. polymerization, oxidative electrocatalysis).

\section{Acknowledgments}

This work was supported by the Australian Research Council (ARC) through an ARC DECRA for S.C. (DE160100732), a
Discovery Projects grant (DP150103065, J.J.G. and S.C.) and an ARC Australian Laureate Fellowship for J.J.G. (FL150100060). The authors acknowledge the use of Curtin University's Microscopy \& Microanalysis Facility, whose instrumentation has been partially funded by the University, State and Commonwealth Governments.

\section{References}

1. A. J. Bard, F. -R. F. Fan, D. T. Pierce, P. R. Unwin, D. O. Wipf, and F. Zhou, Science, 254, 68 (1991).

2. A. Kumar, F. Ciucci, A. N. Morozovska, S. V. Kalinin, and S. Jesse, Nat. Chem., 3, 707 (2011).

3. S. Bergner, P. Vatsyayan, and F. -M. Matysik, Anal. Chim. Acta, 775, 1 (2013).

4. D. Polcari, P. Dauphin-Ducharme, and J. Mauzeroll, Chem. Rev., 116, 13234 (2016).

5. F. Conzuelo, K. Sliozberg, R. Gutkowski, S. Grützke, M. Nebel, and W. Schuhmann, Anal. Chem., 89, 1222 (2017).

6. E. Cortón and S. R. Mikkelsen, Electrochemical Arrays for Bioassay Applications, in: F.-M. Matysik, (Ed.) Trends Bioelectroanal., Springer International Publishing, Cham, 2017, pp. 103.

7. A. J. Bard, F. R. F. Fan, J. Kwak, and O. Lev, Anal. Chem., 61, 132 (1989).

8. D. Momotenko, J. C. Byers, K. McKelvey, M. Kang, and P. R. Unwin, ACS Nano, 9 , 8942 (2015)

9. C. Combellas, M. Fermigier, A. Fuchs, and F. Kanoufi, Anal. Chem., 77, 7966 (2005).

10. M. H. Choudhury, S. Ciampi, Y. Yang, R. Tavallaie, Y. Zhu, L. Zarei, V. R. Goncales, and J. J. Gooding, Chem. Sci., 6, 6769 (2015).

11. Y. Yang, S. Ciampi, Y. Zhu, and J. J. Gooding, J. Phys. Chem. C, 120, 13032 (2016).

12. Y. B. Vogel, N. Darwish, M. B. Kashi, J. J. Gooding, and S. Ciampi, Electrochim. Acta, 247, 200 (2017).

13. B. Y. Chow, C. J. Emig, and J. M. Jacobson, Proc. Natl. Acad. Sci. U. S. A., 106, 15219 (2009)

14. T. L. Rose, D. H. Longendorfer, and R. D. Rauh, Appl. Phys. Lett., 42, 193 (1983).

15. S. -H. Huang, L. -S. Wei, H. -T. Chu, and Y. -L. Jiang, Sensors, 13, 10711 (2013).

16. S. Y. Lim, Y. -R. Kim, K. Ha, J. -K. Lee, J. G. Lee, W. Jang, J. -Y. Lee, J. H. Bae, and T. D. Chung, Energ. Environ. Sci., 8, 3654 (2015).

17. N. Liu, F. Wang, L. Liu, H. Yu, S. Xie, J. Wang, Y. Wang, G. -B. Lee, and W. J. Li, Sci. Rep., 6, 32106 (2016).

18. S. Ciampi, M. James, G. Le Saux, K. Gaus, and J. Justin Gooding, J. Am. Chem. Soc., 134, 844 (2012).

19. L. Zhang, Y. B. Vogel, B. B. Noble, V. R. Gonçales, N. Darwish, A. L. Brun, J. J. Gooding, G. G. Wallace, M. L. Coote, and S. Ciampi, J. Am. Chem. Soc., 138, 9611 (2016).

20. Y. B. Vogel, L. Zhang, N. Darwish, V. R. Gonçales, A. Le Brun, J. J. Gooding, A. Molina, G. G. Wallace, M. L. Coote, J. Gonzalez, and S. Ciampi, Nat. Commun., 8, 2066 (2017).

21. K. -S. Choi, Dalton T,, 5432 (2008).

22. C. -S. Tan, S. -C. Hsu, W. -H. Ke, L. -J. Chen, and M. H. Huang, Nano Letters, 15, 2155 (2015)

23. H. Mistry, A. S. Varela, S. Kühl, P. Strasser, and B. R. Cuenya, Nat. Rev. Mater, 1, 16009 (2016)

24. M. B. Gawande, A. Goswami, F. -X. Felpin, T. Asefa, X. Huang, R. Silva, X. Zou, R. Zboril, and R. S. Varma, Chem. Rev., 116, 3722 (2016).

25. R. Venkatasubramanian, J. He, M. W. Johnson, I. Stern, D. H. Kim, and N. S. Pesika, Langmuir, 29, 13135 (2013). 